\title{
Treatment of keloid scars with intralesional triamcinolone and 5-fluorouracil injections - a randomized controlled trial
}

Hietanen, K. E.

2019-01

Hietanen , K E , Jarvinen , T A , Huhtala , H , Tolonen , T T , Kuokkanen , H O \& Kaartinen , I S 2019 , ' Treatment of keloid scars with intralesional triamcinolone and 5-fluorouracil injections - a randomized controlled trial ' , Journal of Plastic, Reconstructive \& Aesthetic Surgery , vol. 72 , no. 1 , pp. 4-11 . https://doi.org/10.1016/j.bjps.2018.05.052

http://hdl.handle.net/10138/309286

https://doi.org/10.1016/j.bjps.2018.05.052

unspecified

publishedVersion

Downloaded from Helda, University of Helsinki institutional repository.

This is an electronic reprint of the original article.

This reprint may differ from the original in pagination and typographic detail.

Please cite the original version. 


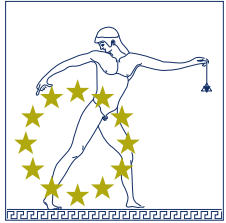

JPRAS

An International journal of Surgical Reconstruction

\title{
Treatment of keloid scars with intralesional triamcinolone and 5-fluorouracil injections - a randomized controlled trial
}

\author{
KE Hietanen ${ }^{a, b, *}$, TA Järvinen ${ }^{a, c}$, H Huhtala ${ }^{d}$, TT Tolonen ${ }^{e}$, \\ HO Kuokkanen ${ }^{f}$, IS Kaartinen ${ }^{a}$
}

\author{
a Department of Musculoskeletal Surgery and Diseases, Tampere University Hospital, Tampere, Finland \\ ${ }^{b}$ Central Finland Health Care District, Jyväskylä, Finland \\ 'School of Medicine, University of Tampere, Tampere, Finland \\ ${ }^{\mathrm{d}}$ Faculty of Social Sciences, University of Tampere, Tampere, Finland \\ e Department of Pathology, Fimlab Laboratories, Tampere University Hospital, Tampere, Finland \\ ${ }^{\mathrm{f}}$ Department of Plastic Surgery, Helsinki University Hospital, Helsinki, Finland
}

Received 14 July 2017; accepted 27 May 2018

KEYWORDS
Keloid;
Scar treatment;
Triamcinolone;
5-fluorouracil;
Immunohistochemistry

\section{KEYWORDS}

Keloid;

Scar treatment;

Triamcinolone;

5-fluorouracil;

Immunohistochemistry

\begin{abstract}
Summary Keloids have high recurrence rates. Current first-line therapy is triamcinolone (TAC) injection, but it has been suggested that approximately $50 \%$ of keloids are steroid resistant. We compared the efficacy of intralesional 5-fluorouracil (5-FU) and triamcinalone injections in a double-blind randomized controlled trial. Forty-three patients with 50 keloid scars were treated with either intralesional TAC or 5-FU-injections over 6 months.

There was no statistically significant difference in the remission rate at 6 months between the 5 -FU and TAC groups (46\% vs $60 \%$, respectively). Local adverse effects were higher in the TAC group compared to the 5-FU group. Occurrence of skin atrophy in TAC group was $44 \%$ and in the 5-FU group $8 \%(\mathrm{p}<0.05)$. Also the occurrence of telangiectasia in the TAC group was $50 \%$ and in the $5-\mathrm{FU} 21 \%(\mathrm{p}<0.05)$. Vascularity of the keloids, assessed by spectral imaging and immunohistochemical staining for blood vessels, after treatment decreased in the TAC group, but not in the $5-\mathrm{FU}$ group $(\mathrm{p}<0.05)$. Fibroblast proliferation evaluated by $\mathrm{Ki}-67$ staining significantly decreased in the TAC group $(\mathrm{p}<0.05)$ but increased in the 5 -FU group $(\mathrm{p}<0.05)$. TAC and 5-FU injections did not differ in their clinical effectivity in this randomized study, but 5-FU injections lead to increased proliferation rate and did not affect vascular density
\end{abstract}

\footnotetext{
*Corresponding author at: Kristiina Hietanen, Central Finland Health Care District, Keskussairaalantie 19, 40620 Jyväskylä, Finland E-mail address: kristiina.hietanen@ksshp.fi (K. Hietanen).
} 
in histological assessment. Due to the greater number of adverse effects observed after TAC treatment, 5-FU injections may be preferable for cosmetically sensitive skin areas.

(C) 2018 British Association of Plastic, Reconstructive and Aesthetic Surgeons. Published by Elsevier Ltd. All rights reserved.

\section{Introduction}

Treatment of keloid scars is challenging for clinicians. Despite several reported strategies, no standard treatment protocol exists. ${ }^{1,2}$ Triamcinolone acetate (TAC) injections are considered as the first treatment option for keloids. ${ }^{1}$ The efficacy of TAC in keloid treatment has been demonstrated in clinical trials already in the 1960 s and $1970 \mathrm{~s}^{3-5}$ Based on these earlier trials, it has been estimated that TAC shows clinical effect in the majority of treated keloids. ${ }^{6}$ However, more recent data suggests that up to $50 \%$ of keloids do not respond to steroid injections. ${ }^{7,8}$ Furthermore, 9-50\% of keloids that initially respond to TAC treatment ultimately relapse. ${ }^{7}$ In addition to quite high proportions of keloids either not responding to TAC or developing recurrence, approximately half of the TAC treated keloids are also associated with side effects such as subcutaneous atrophy and telangiectasia. ${ }^{1}$ Thus new therapeutic options, among them antineoplastic agent 5-fluorouracil (5-FU) have emerged as a potential option to improve treatment efficacy without adverse effects. 5-FU is a fluorinated pyrimidine antimetabolite that inhibits fibroblast proliferation. ${ }^{1,9}$ A possible mechanism of action of 5-FU is the selective blockage of collagen synthesis by keloid fibroblasts. ${ }^{9,10}$

Preliminary studies comparing 5-FU with TAC have been limited and failed to provide conclusive evidence of efficacy. ${ }^{11,12}$ The aim of this study was to compare the efficacy and possible side effects of TAC and 5-FU injections for the treatment of keloids in a double-blind, randomized, controlled trial.

\section{Patients and methods}

The study was approved by the Ethics Committee of the Pirkanmaa Health Care District and recorded in the prospective clinical studies database: ClinicalTrials.gov (\#NCT02155439).

Initially, 105 patients with previously treated keloid scars were seen at the outpatient clinic. A total of 43 patients with 50 active and symptomatic keloid scars requiring treatment were enrolled and randomized into two groups. All patients were counseled about the study design and given the option to enroll. After initial screening and a cooling off period, patients who enrolled provided consent to the study. Some keloids were treated for first time (Table 1). A keloid was defined as a scar growing outside the boundaries of the original wound without signs of resolution over 3 years. Inclusion criteria of this study were: any patient over 18 years of age undergoing keloid treatment in Tampere University Hospital and suitable for treatment with drug injections (Supplemental Figure Consort 2010 flow diagram). The exclusion criteria of this study excluded: patients under the age of 18 , pregnancy or planned pregnancy, lactation, renal failure, liver dysfunction, hematological disease or bone marrow suppression, systemic or local infection. The causes and anatomical sites of the keloids are shown in Table 1.

A permutated-block randomization with closed envelopes was used to ensure equal group sizes, leading to 2 groups, each with 25 keloid scars. Each randomized selection of envelope was performed blindly by the same plastic surgeon (the senior author) who also performed the drug injections without revealing the patient allocation (i.e. drug treatment to the patients). The response to therapy was assessed independently by another plastic surgeon (the first author). The patient and observer were blinded at the time the outcome was assessed. Follow up was performed at 6 months. By that time all of the data had been analyzed in single blinded fashion. The plastic surgeon performing the injections did not perform any analysis of collected data.

The patients were treated with intralesional injections of either TAC or 5-FU. All the patients were reviewed in for follow up assessment a total of five times (once every 3-4 weeks until week 12 and at 6 months).

The injections were given during the first three visits by the same senior plastic surgeon, who alone knew the result of randomization. The injections were given according to the published recommendations. Injection technique was equal as described in previous papers, i.e. under local anesthesia, either TAC or 5-FU was injected from several directions directly into the keloid with care taken not to inject under the keloid mass or too close to epidermis to avoid unnecessary local side effects. The injectable was introduced into the scar until blanching occurred. For patients who responded well and did not need three injections, control visits were carried out without further injections (Table 2). There were no placebo injections, i.e. the patients were randomized into two groups having either TAC or 5-FU injections.

For TAC injections, Lederspan $®$ (Haupt Pharma Wolfratshausen $\mathrm{GmbH}$, Germany) $20 \mathrm{mg} / \mathrm{ml}$ mixed 1:1 with lidocaine $10 \mathrm{mg} / \mathrm{ml}$ (Orion Pharma, Finland) was used. For 5-FU injections, 5-Fluorouracil Accord $₫$ (AccordHealthCare Ltd North Harrow, UK) was used at a concentration of $50 \mathrm{mg} / \mathrm{ml}$. Both groups received local anesthesia with lidocaine first. 5 -FU cannot be mixed with lidocaine, as it is a cytostatic and prepared in the hospital pharmacy. However, TAC can be uniformly administered mixed with a local anesthetic, hence we used it in this way.

A blinded observer (the first author) assessed the keloids using a validated scar scale, the Patient and Observer Scar Assessment Scale (POSAS) and objective assessment was performed with a spectrocutometer camera. ${ }^{13}$ Keloids were separately assessed and evaluated for evidence of skin atrophy and telangiectasia as adverse effects after both treatments. Each patient filled out the patient component 
Table 1 Demographics.

\begin{tabular}{|c|c|c|c|c|}
\hline Characteristic & & TAC $(n=25)$ & $5-F U(n=25)$ & $\mathrm{P}$ \\
\hline \multirow[t]{4}{*}{ Age, years } & & & & $\overline{N D^{* *}}$ \\
\hline & Median & 40 & 43 & \\
\hline & Mean & 41 & 46.96 & \\
\hline & Range & $18-77$ & $21-81$ & \\
\hline \multirow[t]{3}{*}{ Sex, $n(\%)$} & & & & $N D^{*}$ \\
\hline & Male & 11 (44\%) & $15(60 \%)$ & \\
\hline & Female & $14(56 \%)$ & $10(40 \%)$ & \\
\hline \multicolumn{5}{|l|}{ Anatomic } \\
\hline \multirow[t]{5}{*}{ Location n (\%) } & & & & $N D^{*}$ \\
\hline & Chest & $6(24 \%)$ & $9(36 \%)$ & \\
\hline & Shoulder & $6(24 \%)$ & 6 (24\%) & \\
\hline & Upper back & $10(40 \%)$ & $1(4 \%)$ & \\
\hline & Abdomen & $3(12 \%)$ & $4(16 \%)$ & \\
\hline \multirow[t]{8}{*}{ Etiology } & & & & $N D^{*}$ \\
\hline & Surgery & $13(52 \%)$ & 12 (48\%) & \\
\hline & Acne & $7(28 \%)$ & $6(24 \%)$ & \\
\hline & Trauma & $2(8 \%)$ & $4(16 \%)$ & \\
\hline & Unknown & $2(8 \%)$ & $1(4 \%)$ & \\
\hline & Vaccination & $1(4 \%)$ & $0(0 \%)$ & \\
\hline & Burn injury & $0(0 \%)$ & $1(4 \%)$ & \\
\hline & Piercing & $0(0 \%)$ & $1(4 \%)$ & \\
\hline \multirow[t]{5}{*}{ Previous treatments } & & & & $N D^{*}$ \\
\hline & None & $8(32 \%)$ & $8(32 \%)$ & \\
\hline & $T A C$ & $15(60 \%)$ & $15(60 \%)$ & \\
\hline & $5-F U$ & 1 (4\%) & $1(4 \%)$ & \\
\hline & Radiation and surgery & $1(4 \%)$ & $1(4 \%)$ & \\
\hline \multirow[t]{4}{*}{ Area $(\mathrm{mm} 2)$} & & & & $N D^{* *}$ \\
\hline & Median & 978 & 799 & \\
\hline & Mean & 1439 & 1473 & \\
\hline & Range & $169-6365$ & $144-7043$ & \\
\hline $\begin{array}{l}\text { ND (No Difference) } \\
* \text { Pearson chi-square- } \\
* * \text { Mann-Whitney U te }\end{array}$ & & & & \\
\hline
\end{tabular}

Table 2 Results: remission rate, adverse effects and number of given injections.

\begin{tabular}{|c|c|c|c|c|c|c|}
\hline & & \multicolumn{2}{|c|}{ TAC $n=25$} & \multicolumn{2}{|c|}{$5-F U n=24$} & \multirow[t]{2}{*}{$\mathrm{p}^{*}$} \\
\hline & & $n$ & $\%$ & $\mathrm{n}$ & $\%$ & \\
\hline \multicolumn{7}{|l|}{ Remission } \\
\hline & & 15 & 60 & 11 & 46 & $>0.05$ \\
\hline \multirow{4}{*}{ Number of injections $\mathrm{n}(\%)$} & & & & & & $>0.05$ \\
\hline & 1 & 2 & 8 & 1 & 4 & \\
\hline & 2 & 10 & 40 & 10 & 42 & \\
\hline & 3 & 13 & 52 & 13 & 54 & \\
\hline \multicolumn{7}{|l|}{ Adverse effects } \\
\hline & Skin atrophy & 11 & 44 & 2 & 8 & $<0.05$ \\
\hline & Telangiectasia & 14 & 50 & 5 & 21 & $<\underline{0.05}$ \\
\hline
\end{tabular}

* chi-square test

of the POSAS scale at each visit. With spectrocutometry, the estimated hemoglobin concentration of the keloid was calculated as described in previous study. ${ }^{14}$

At the first three visits, a $3 \mathrm{~mm}$ punch biopsy was obtained from the active border of the keloid for histological and immunohistochemical analyses. The first biopsy was obtained before any treatment was given. The second and third biopsies were obtained after the first and second injections at 4 and 8 weeks respectively. Blood vessel density was analyzed by counting blood vessels (CD31) and fibroblast proliferation was evaluated by Ki-67 staining from the biopsy samples.

The primary endpoint of the study was remission of the keloid at 6 months after treatment; secondary endpoints included the POSAS score, local adverse effects, blood vessel density, estimated change in the hemoglobin concentration 
and fibroblast proliferation. The remission of the keloid was defined clinically by the blinded observer as flattening of the keloid to the degree where no further injections were feasible or needed. Same blinded observer did the conclusions of possible occurred side effects, skin atrophy and telangiectasia.

\section{Immunohistochemical (IHC) staining}

Biopsy samples of keloids were collected and fixed with 4\% paraformaldehyde and embedded in paraffin according to standard protocols. Hematoxylin/eosin staining and immunohistochemical staining (IHC) was performed from 6- $\mu \mathrm{m}$ thick paraffin sections as previously described. ${ }^{15,16}$ Briefly, Formalin fixed, paraffin-embedded tissue sections were deparaffinized, processed with appropriate antigen retrieval solution (10 mM Tris-HCL, $1 \mathrm{mM}$ EDTA, $\mathrm{pH}$ 9), incubated with the blocking reagent $(10 \%$ normal goat serum, $5 \%$ non-fat milk and 5\% BSA), and endogenous peroxidase activity was suppressed with hydrogen peroxide. Tissue sections were incubated with the primary antibody overnight at $4{ }^{\circ} \mathrm{C}$. The rat anti-CD31 (clone 550,274) and rat anti-Ki67 (clone M7249 TEC-3) antibodies were used for IHC (both BD Pharmingen, Oxford, UK) followed by the appropriate horseradish peroxidase-conjugated (HRP) secondary antibodies. ${ }^{15}$ The blocking reagents used for IHC were S2O23 REAL and S0809 Antibody Diluent (DakoCytomation). The peroxidase reactive chromogen used was 3.3 diaminobenzidine (DAB; K3465,DAKO, Agilent Technologies). Each staining experiment included sections stained without primary antibody as negative controls.

\section{Quantitative analysis of immunostaining and histochemical staining}

Image analysis as well as the quantification of histological and immunohistochemical (IHC) parameters were done using Spectrum digital pathology system (Aperio Technologies). ${ }^{15-17}$ Briefly, all histological slides were scanned to digital microscope using the Aperio ScanScope ${ }^{\circledR}$ CS and XT systems (Aperio Technologies Inc., Vusta CA, USA). ${ }^{17}$ Slides were viewed and analysed remotely using desktop personal computers employing the web-based ImageScope ${ }^{T M}$ viewer. The Spectrum digital pathology system analysis algorithm package and Image Scope analysis software (version 9; Aperio Technologies Inc.) was used to quantify the IHC signal. The Aperio-software separates the signal from the chromogen and hematoxylin/eosin by a color deconvolution method to identify negative and positive cells/areas. Custom made algorithms were generated for each respective staining. The software was used to calculate the area of positive and negative staining, the average positive intensity (optical density), and graded as weak (1+), medium $(2+)$, and strong $(3+)$ positive staining. ${ }^{17}$ The quantified histochemical analysis of CD31- and Ki-67stainings were performed according to the protocols used to establish these algorithms for each type of stain. ${ }^{15,17}$ At least 5000 cells (or entire keloid biopsy for CD31) were counted for each measurement from each sample. All evaluations were possible were performed in an unbiased blinded fashion i.e, the examiner did not know which group (TAC/5-FU) the specimens belonged.

\section{Statistical analysis}

Statistical analyses were performed with SPSS version 21.0 software IBM SPSS Statistics for Windows. (Version 21.0. Armonk, NY: IBM Corp.)

For comparison of patient characteristics between the TAC and 5-FU group, the chi-square (discrete variables) test and Mann-Whitney $U$ test (continuous variables) were used. The frequency of side effects was analyzed with a chisquare test. A paired $t$-test was used for comparisons between the baseline and post-treatment values of CD31, Ki67 , hemoglobin concentration and POSAS scores. A P-value less than 0.05 was considered statistically significant.

\section{Results}

One patient dropped out from the 5-FU group after the first visit, because the patient decided against keloid treatment. Also, one patient with two keloids in the 5-FU group discontinued the treatment due to a local allergic reaction to the second 5-FU injection, comprising of urticaria and local blistering that resolved after a day and required no additional treatment. For the final analysis, the 5-FU group comprised of 24 keloids and TAC group comprised of 25 keloids. The clinical characteristics of the patients in the two groups were comparable (Table 1). In the TAC group a total of 61 injections were given and the mean dosage was $8.4 \mathrm{mg}$ ( $\pm 5.4 \mathrm{mg}$, range $1-35 \mathrm{mg})$ and the mean number of injections were 2.4 (range 1-3), whereas in the 5-FU group a total of 60 injections were given with a mean dosage of $25 \mathrm{mg}$ ( $\pm 13 \mathrm{mg}$ range $7.5-50 \mathrm{mg})$ and the mean number of injections were 2.4 (range 1-3).

Treatment efficacy did not differ significantly between the two groups. The remission rate at 6 months was $46 \%$ in the $5-\mathrm{FU}$ group compared to $60 \%$ in the TAC group ( $>>0.05)$. Clinically, flattening and softening of the keloids was noticed after both 5-FU and TAC injections in keloids responding to the treatment (Figure 1). Skin atrophy was seen in $44 \%$ of the patients in the TAC group and in $8 \%$ of the patients in the 5-FU group (Table 2). The difference was statistically significant $(p<0.05)$. Also telangiectasia was seen in $50 \%$ of the patients in the TAC group and in $21 \%$ of the patients in the 5-FU group ( $<<0.05)$. The evaluation of side effects was performed by unmagnified clinical observation for both telangiectasia and skin atrophy. The POSAS scores (Observer and Patient) decreased in both the 5-FU and the TAC groups during the 6 months follow-up (Figure 2). The POSAS evaluation (observer and patient) between baseline and 6 months was improved $(p<0.05)$ in both groups, however there was no difference seen between the two treatment groups. The estimated hemoglobin concentration had decreased at 6 months compared to the baseline in the TAC group $(0.17 \pm 0.18$ and $0.10 \pm 0.18, p<0.05)$. In the 5 -FU group, the estimated haemoglobin concentration at 6 months did not significantly change from the baseline value $(0.13 \pm 0.10$ and $0.10 \pm 0.09,(p>0.05)$ (Figure 3$)$. The blood vessel density (defined as CD31-positive area) 


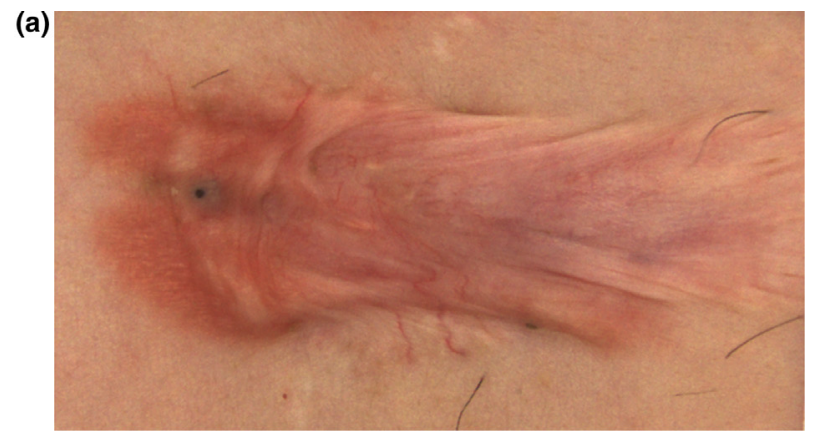

(b)

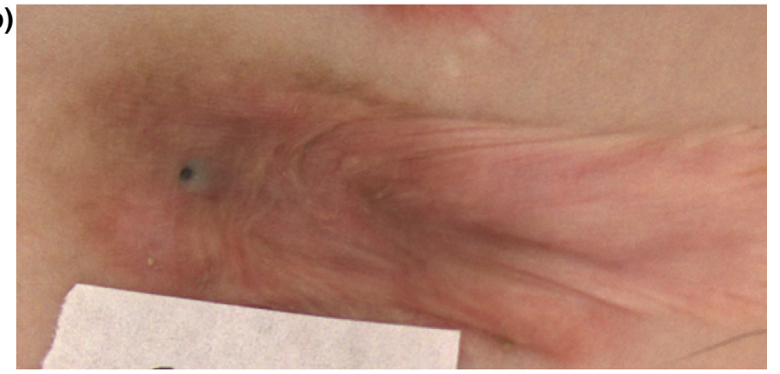

(c)

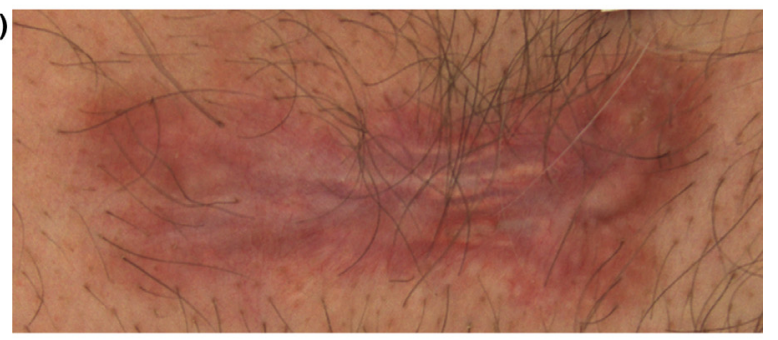

(d)

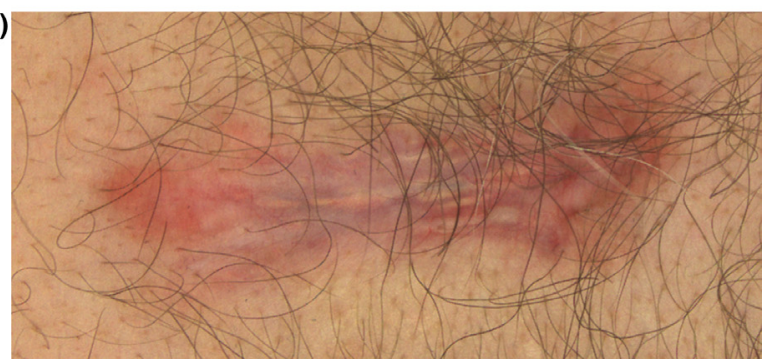

Figure 1 Example of a keloid treated with 5-FU injections before (a) and after (b) treatment and another keloid treated with TAC injections before (c) and after (d) treatment. Both keloids shown here were in remission in the end of the 6 months followup. The small comedo in $A$ and $B$ is unrelated to treatment.

decreased in the entire 49- keloid group, but in the 5-FU group, the change was not significant $(p>0.05)$ (Figures 4 and 5). In the TAC group, the blood vessel density significantly decreased from baseline value $(p<0.05)$. The reduction in blood vessel density measured supported the findings that a reduction of estimated hemoglobin concentration in keloids analyzed with spectrocutometry (Figures 3-5).

Ki-67 values were analyzed from the whole keloid area reticular dermis excluded. The mean proliferation rate were 9.9\% (range 3.3-16.3) at baseline and $12.1 \%$ (range 6.4-21.2) after treatment in the 5-FU group whereas the mean proliferation rates in the TAC group were $11.3 \%$ (range 1.925.3) at baseline and $7.9 \%$ (range 1.7-24.7) after treatment. The difference before and after treatment was (a) Observer POSAS

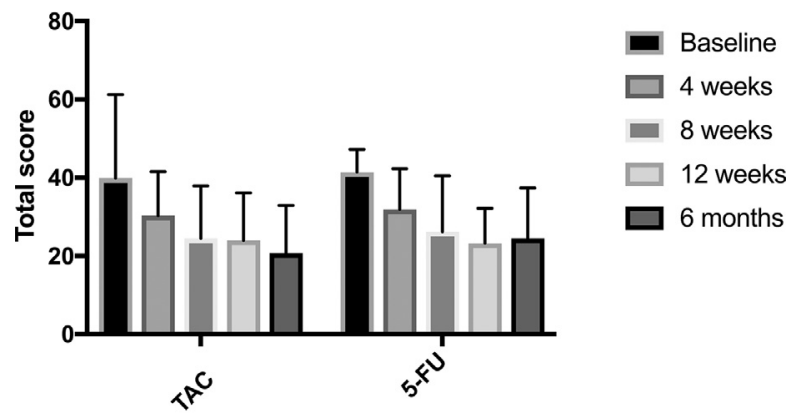

(b) Patient POSAS

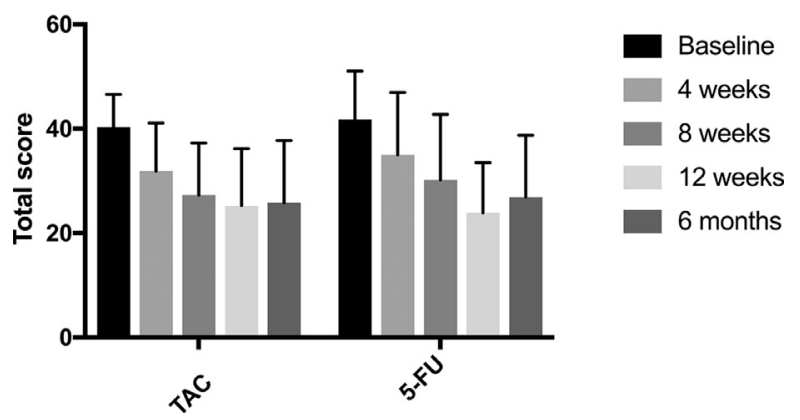

Figure 2 (A) POSAS Observer scores in the 5-FU and TAC groups. (B) POSAS Patient scores in the 5-FU and TAC groups.

\section{Oxyhemoglobin concentration}

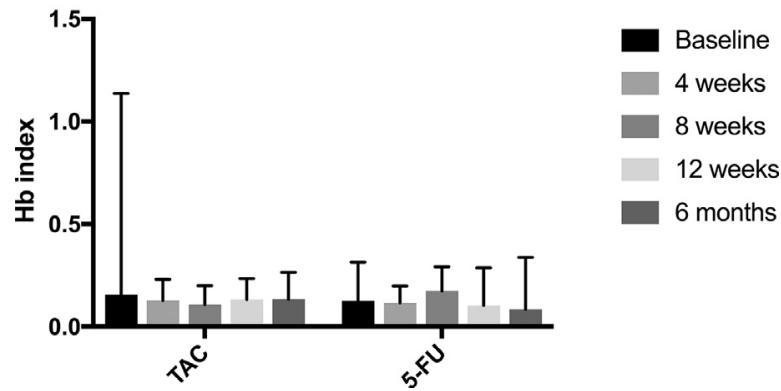

Figure 3 Hemoglobin $(\mathrm{Hb})$ concentration at each visit, measured from the entire scar area by means of standardized digital imaging and spectrocutometry. $\mathrm{Hb}$ concentration in the scar is shown as blood haemoglobin index.

statistically significant in both groups, but in the 5-FU group proliferation rate was increased by the treatment $(p<0.05)$ and in the TAC group it decreased $(\mathrm{p}<0.05)$ (Figures 5 and 6).

\section{Discussion}

Treating keloids with TAC injections has been common practice since the 1970s. ${ }^{1,3,4}$ Keloid treatment with 5-FU, however, is still considered as experimental although few randomized controlled trials have studied the effects of 5-FU treatment on keloid scars. Nanda et al. and Kontochristopoulos et al. published a series of patients treated 


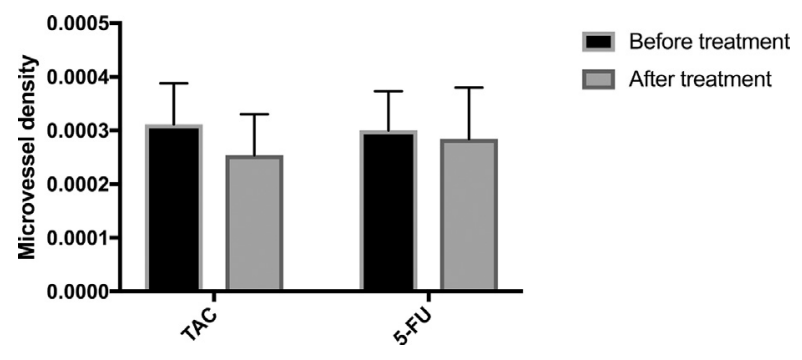

Figure 4 Blood vessel density before and after treatment in 5FU and TAC groups. The keloids were treated with either TAC or 5 -FU injections in a randomized controlled trial. The blood vessels were determined from the tissue biopsies obtained from the keloids before and after the treatment. Tissue sections were stained by antibody against endothelial cells (CD31) and quantitative digital pathology analysis of scanned slides was performed. The results are expressed as the area covered by the blood vessels in the total keloid area in the biopsy.

with 5-FU alone. ${ }^{18,19}$ Both of these studies reported favorable outcomes, with a 70 to $95 \%$ success rate. Neither of these studies, however, was a randomized controlled trial. Sadeghinia et al. conducted a randomized controlled trial with 44 patients comparing the efficacy of 5-FU and TAC injections. ${ }^{11}$ Their study was similar to ours in terms of sample size, injection rate and concentrations and dosages used. Sadeghinia et al. graded the overall improvement using a 5 -point scale. ${ }^{11}$ Both patient reported outcome and assessment by the observer indicated that 5-FU injections produced significantly better results compared to TAC injections. We found no significant difference in the efficacy of the two treatments in the present study. We observed a positive response in both the TAC and the 5-FU groups, and the improvement between the baseline and 6 months that was statistically significant in both groups. However, remission rate after 5-FU treatment was lower than in previous studies mentioned above and although there was a significant effect of 5-FU injections, less than half of the patients in this study were in remission after 5-FU treatment.

In our study permanent local side effects were more common in the TAC group than in the 5-FU group. The side effects of TAC injections have been reported also in previous trials. Sadeghia et al. reported no side effects in either group; Nanda et al. reported ulceration in $21.4 \%$ and burning sensation in $7.1 \%$ at the 5 -FU treatment sites. ${ }^{11,18}$ However, TAC treatment was associated with significant local side effects in other reports. ${ }^{1,2,20,21}$ Fitzpatrick and Haurani et al. reported their experiences with 5-FU injections for hypertrophic scarring, and observed no systemic or local adverse effects. ${ }^{9,22}$ On the other hand Kontochristopoulos et al. reported that all 20 of their patients found the injections painful and 30\% presented with superficial ulcerations in their keloids after 5-FU injections. ${ }^{19}$ The same study showed that all 20 patients developed hyperpigmentation at the treatment sites, but this did not cause any significant cosmetic morbidity and gradually subsided. ${ }^{19}$ Srivastava et al. studied TAC, 5-FU and their combination in keloid treatment in a randomized parallel group study with $60 \mathrm{pa}$ tients. ${ }^{12}$ They stated that TAC, 5-FU and their combination are all effective in keloid treatment, but the combination
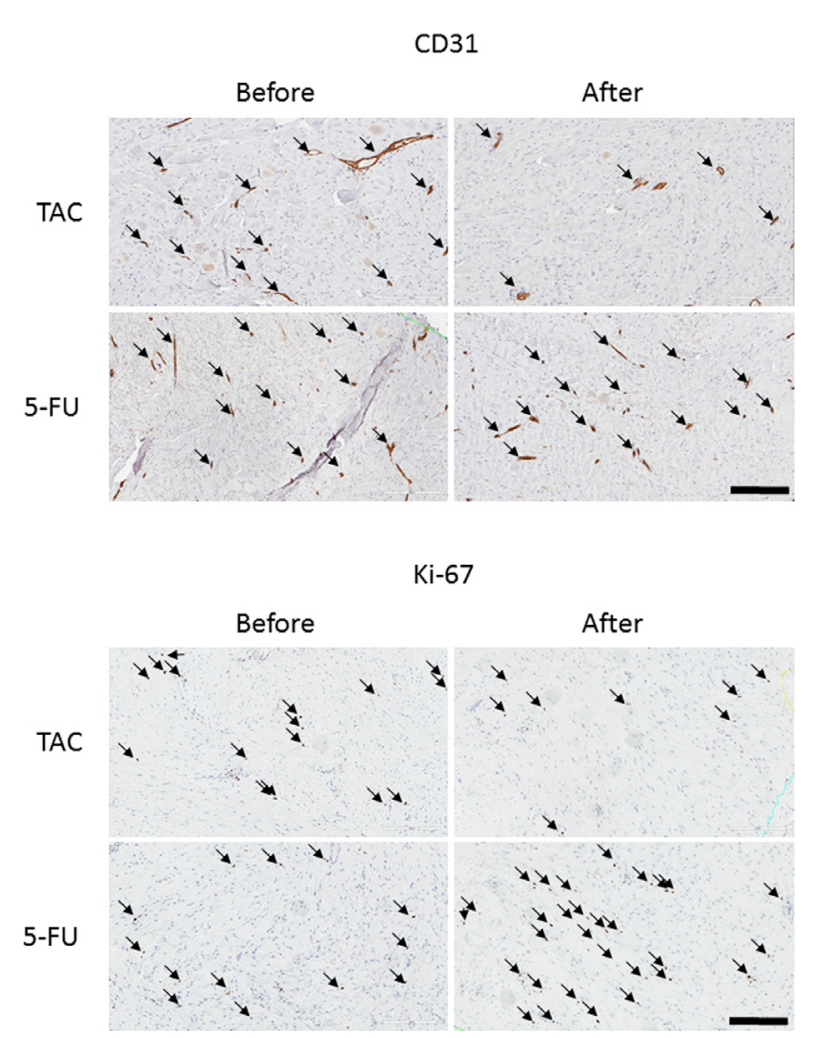

Figure 5 Immunohistochemical stainings for CD31 and Ki-67 before (left column) and after (right column) treatment in 5FU and TAC groups. Positive endothelial cells (CD31) and stromal cell nuclei (Ki-67) marked by arrowheads. Vascular density measured by CD31 decreased significantly in the TAC group but not in the 5-FU group. Proliferation rate measured by Ki-67 decreased significantly in the TAC group and increased in the 5-FU group. The scale bar in the lower right corner measures 200 $\mu \mathrm{m}$.

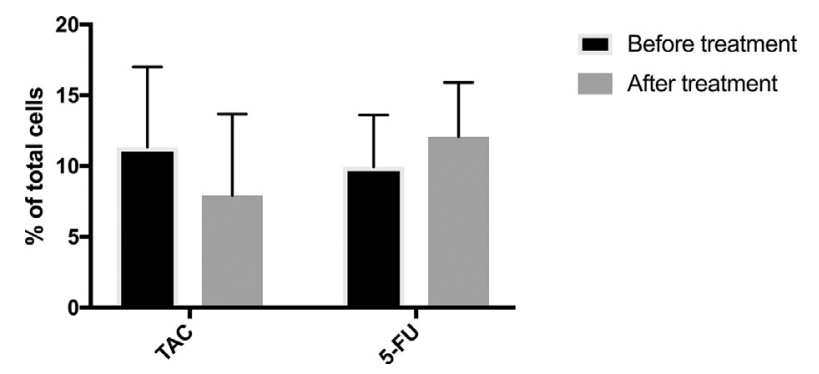

Figure 6 Cell proliferation rates at baseline and posttreatment. The keloids were treated with either TAC or 5-FU injections in a randomized controlled trial. Skin biopsies were obtained from the edges of keloids before and after the treatment. Biopsies were fixed and processed for IHC staining of proliferating nuclei by using an antibody against Ki67, a wellestablished proliferation marker. Quantitative digital pathology analysis of scanned slides was performed. The results are expressed as the percentage of proliferating cells in total keloid cell population. 
treatment of TAC and 5-FU was superior in efficacy and incidence of side effects. ${ }^{12}$ Srivastava et al. reported skin ulceration in 9 patients of their 20 patients in the 5-FU group ${ }^{12}$. Contrary to their findings our side effect profile was low. In our study, one patient developed a local allergic reaction and skin blistering after the second injection of 5-FU, which resulted in discontinuing the treatment. This was the only immediate local side effect in this study and it is not clear why this occurred and whether it was the cytotoxic effect that caused the ulceration.

Three patients exhibited hyperpigmentation in the 5-FU group. The TAC group presented with significantly more permanent local adverse effects $44 \%$ with skin atrophy and $50 \%$ with telangiectasia compared to $8 \%$ and $21 \%$ in the 5 -FU group respectively. This observation may support the role of 5-FU over TAC especially in cosmetically important areas, where local adverse effects may lead to considerable aesthetic problems. However clear conclusion cannot be drawn from this limited study. A greater number of patients recruited to this study would be needed to provide a more accurate side effect profile. Another trial comparing TAC and $\mathrm{TAC}+5-\mathrm{FU}$ may provide some insight into the added efficacy combinatorial therapy may offer.

We have shown that the vascularity of a keloid may be reduced by TAC injections as shown by decreased blood vessel density and estimated keloid hemoglobin concentrations through spectrocutometry assessment. Increased vascularity is reported in both keloids and hypertrophic scars and is an indicator of ongoing metabolic scar activity. ${ }^{23,24}$ Our finding that hemoglobin concentration in keloids defined by spectrocutometry correlates with actual blood vessel density may further encourage the use of spectrocutometry as an objective assessment method in scar treatment. In our study vascular density in keloids were not significantly changed following 5-FU injections. Also Kontochristopoulos et al. found vascularity to be slightly decreased after 5-FU treatment. ${ }^{19}$ Their finding, however, was not verified with IHC. Studies have shown TAC treatment may decrease sprouting angiogenesis. ${ }^{25,26}$ Further studies need to be performed to validate the sensitivity and specificity of these modalities in scar outcomes.

Kontochristopoulos et al. also studied the proliferation rate of fibroblasts with $\mathrm{Ki}-67 .{ }^{19}$ They reported a decrease (1-5\% to $0.5-2 \%)$ in $\mathrm{Ki}-67$ in all their 10 cases after 6 5-FU injections treatment. However, we examined 24 5-FU treated keloids before and after 1-3 injections treatment and found that the level of $\mathrm{Ki}-67$ increased in the 5-FU group, and decreased in the TAC group. This may warrant further investigation into the action pathways of 5-FU in keloid treatment.

The weakness of our study is there was a short 6 months follow-up time, which is not long enough to observe for keloid recurrence, which can occur as late as 24 months or more after treatment. More modalities of assessment would have strengthened this study however we do provide some preliminary data that suggest the efficacy of TAC and 5-FU are similar but not dramatically different. One can see that these treatments usually show some benefit after 3-4 injections, however if no response is seen, it is not recommended to continue with the same line of treatment. Where benefits were not seen, patients from this study went on to have surgery and radiotherapy, and some had further substance injections (TAC, 5-FU or verapamil) as well as pressure garment or silicone gel sheeting. This is a limited study for this reason and does not aim to challenge the efficacy of other well studied modalities but focuses on comparing TAC to 5-FU in there role as early minimally invasive treatment modalities.

\section{Conclusion}

5-FU injections are effective in some patients when assessed clinically, but showed no clear clinical benefit over TAC. TAC injections may lead to better remission rate than 5-FU injections, with slightly more local adverse effects such as skin atrophy and telangiectasia, but there was no statistically significant difference in this study in remission rate between the two therapies. A significant number of patients do not respond to either of the two injection treatments, hence progressing them through to other strategies would seem reasonable after a six month trial of injectable therapies.

\section{Acknowledgments}

We thank Marianne Karlsberg and Marja-Leena Koskinen for performing the immunohistologic stainings and Mrs. Guillermina Garcia (Sanford Burnham Prebys Medical Discovery Institute, La Jolla, CA, USA) for her technical expertise and help with quantitative microscopy. We also thank $\mathrm{Dr}$ Tec Petri Välisuo in department of Electrical and Energy Engineering of University of Vaasa, performing the analysis with spectrocutometer camera.

\section{Author disclosure and funding}

This work was funded by the Academy of Finland, Päivikki and Sakari Sohlberg Foundation, Vappu Uuspää foundation, Instrumentarium Research Foundation, Orion Research Foundation and Competitive State Research Financing of the Expert Responsibility area of Tampere University Hospital, Grant 9R025 and 9S025. The authors have no conflicts of interest to declare and no financial interests exist.

\section{Supplementary materials}

Supplementary material associated with this article can be found, in the online version, at doi:10.1016/j.bjps.2018.05. 052.

\section{References}

1. Al-Attar A, Mess S, Thomassen JM, et al. Keloid pathogenesis and treatment. J Plast Reconstr Surg 2006;117:286-300.

2. Chike-Obi CJ, Cole PD, Brisset AE. Keloids: pathogenesis, clinical features, and management. Seminars Plast Surg 2009;23:178-84.

3. Hollander A. Intralesional injections of triamcinoloneacetonide: a therapy for dermatomes. Antibiot Med Clin Ther $1961 ; 8: 78$. 
4. Ketchum LD, Robinson DW, Masters FW. Follow-up on treatment of hypertrophic scars and keloids with triamcinolone. J Plast Reconstr Surg 1971;48:256-9.

5. Murray RD. Kenalog and the treatment of hypertrophied scars and keloids in negroes and whites. J Plast reconstr surg $1963 ; 31: 275$

6. Mustoe TA, Cooter RD, Gold MH. International clinical recommendations on scar management. J Plast Reconstr Surg 2002;110:560.

7. Ud-Din S, Bowring A, Derbyshire B, Morris J, Bayat A. Identification of steroid sensitive responders versus non-responders in the treatment of keloid disease. Arch Dermatol Res 2013;305:423-32.

8. Larrabee WF, East CA, Jaffe HS. Intralesional interferon gamma treatment for keloids and hypertrophic scars. Arch Otolaryngol Head Neck surg 1990;116:1159.

9. Haurani MJ, Foreman K, Yang JJ, Siddiqui A. 5-fluorouracil treatment of problematic scars. J Plast Reconstr Surg 2009; 123:139-48.

10. Bulstrode NW, Mudera V, McGrouther DA. 5-fluorouracil selectively inhibits collagen synthesis. J Plast Reconstr Surg 2005; 116:209.

11. Sadeghinia A, Sadeghinia S. Comparison of the efficacy of intralesional triamcinolone acetonide and 5-fluorouracil tattooing for the treatment of keloids. Dermatol Surg 2012;38:104-9.

12. Srivastava S, Patil A, Prakash C, Kumari H. Comparison of intralesional triamcinolone acetonide, 5-Fluorouracil, and their combination for the treatment of keloids. Adv Wound Care 2017;6:11.

13. Kaartinen IS, Valisuo PO, Alander JT, Kuokkanen HO. Objective scar assessment-a new method using standardized digital imaging and spectral modelling. Burns 2011;37:74-81.

14. Kaartinen IS, Valisuo PO, Bochko V, Alander JT, Kuokkanen HO. How to assess scar hypertrophy-a comparison of subjective scales and Spectrocutometry: a new objective method. WRR 2011;19:316-23.
15. May U, Prince S, Vähätupa M, et al. Resistance of R-Ras knockout mice to skin tumour induction. Sci Rep 2015;5:11663.

16. Vähätupa M, Aittomäki S, Martinex, et al. T-cell-expressed proprotein convertase FURIN inhibits DMBA/TPA-induced skin cancer development. Oncolmmunology 2016;5:1245266.

17. Järvinen TA, Ruoslahti E. Target-seeking antifibrotic compound enhances wound healing and suppresses scar formation in mice. Proc Natl Acad Sci USA 2010;107:21671-6.

18. Nanda S, Reddy BS. Intralesional 5-fluorouracil as a treatment modality of keloids. Dermatol Surg 2004;30(56-7):54-6.

19. Kontochristopoulos G, Stefanaki C, Panagiotopoulos, et al. Intralesional 5-fluorouracil in the treatment of keloids: an open clinical and histopathologic study. J Am Acad Dermatol 2005;3(52):474-9.

20. Ogawa R. The most current algorithms for the treatment and prevention of hypertrophic scars and keloids. J Plast Reconstr Surg 2010; 125:557-68.

21. Hietanen K, Välisuo $P$, Kuokkanen $H$, Kaartinen I. Long-term results of intralesional triamcinolone acetonide injections in keloid treatment. J Clin Trials 2016;6:4.

22. Fitzpatrick RE. Treatment of inflamed hypertrophic scars using intralesional 5-FU. Dermatol Surg 1999;25:224-32.

23. Manuskiatti W, Fitzpatrik RE, Goldman MP. Energy density and numbers of treatment affect response of keloidal and hypertrophic sternotomy scars to the $585-\mathrm{nm}$ flashlamp-pumped pulsed-dye laser. J Am Acad Dermatol 2001;45:557565.

24. Chan HH, Wong DSY, Ho WS. The use of pulsed dye laser for prevention and treatment of hypertrophic scars in Chinese persons. Dermatol Surg 2004;30:987-94.

25. Hochman B, Locali RF, Matsuoka PK. Intralesional triamcinolone acetonide for keloid treatment: a systematic review. Aesth Plast Surg 2008;32:705-9.

26. Leventhal D, Furr M, Reiter D. Treatment of keloids and hypertrophic scars. Arch Facial Plast Surg 2006;8:362-8. 